\title{
Complex Coordination Control with Entire Power Limitation and Partial Temperature Priority of Building-Multi Air-conditioning Facilities for Real-Time Pricing
}

\author{
Yoshifumi Aoki* \\ Chuzo Ninagawa $^{* a)}$ \\ Takashi Inaba** \\ Hiromichi Ito* \\ Junji Morikawa* \\ Seiji Kondo** \\ Non-member \\ Non-member \\ Non-member
}

\begin{abstract}
Real-time pricing is one of the primary features of demand response schemes for the future smart grids. From the demand side's point of view, coordination of load management conforming to the rapid power price changes is necessary. This study attempts to optimize a number of multi-unit-type air-conditioning facilities installed in an office building by using a complex evaluation function, which consists of power cost, room temperature comfort terms with priority, and total power consumption. Since an actual experiment lacks reproducibility, simulation experiments with different electricity pricing patterns are carried out. An original air-conditioner emulating model is developed and used to conduct the simulation experiments.
\end{abstract}

Keywords: real-time pricing, multi-unit-type building air-conditioner, simulated annealing

\section{Introduction}

Fast automated demand response (FastADR) may be achieved in future smart grids through real-time pricing (RTP) in which the electricity charge is greatly changed over intervals of several tens of minutes ${ }^{(1)(2)}$. Next-generation building energy management systems (BEMSs) may require applicability to FastADR. Of consumer electric facilities, air conditioning facilities for commercial use and office building multi-air conditioning facilities in which controllable power consumption is largely installed and installed at great volume could be a promising resource of FastADR ${ }^{(3)-(10)}$.

Air conditioning control in an RTP environment must take into account not only electricity charges but also air conditioning comfort. For example, under the circumstance of which the electricity unit price changes at a factor of 10 at every time slot of approximately 10 minutes, it would be conceivable to apply electric power limits to each air conditioning facility when a high electricity charge slot approaches, in order to attempt to minimize the electricity charges. And then the electric power limits will be relaxed when a low electricity charge slot approaches, in order to restore comfort. However, there is a tradeoff between air conditioning electric power and comfort maintenance, so an algorithm is needed that plans a permutation of a power limitation command value so that the tradeoff is adjusted over the total

a) Correspondence to: Chuzo Ninagawa. E-mail: ninagawa@ gifuu.ac.jp

* Faculty of Engineering, Gifu University

1-1, Yanagido, Gifu-city, Gifu 501-1193, Japan

** Mitsubishi Heavy Industries Thermal Systems, Ltd.

3-1, Asahi, Nishibiwajimacho, Kiyosu 452-8561, Japan course of time.

Research has previously been performed on variations in air conditioning electricity charges in units of several hours ${ }^{(11)(12)}$. Research on the tradeoff between RTP handling and comfort ${ }^{(13)}$ and on RTP dynamic handling ${ }^{(14)}$ has also started to appear. However, although there are some studies related to electricity charges and comfort with multi-unit building air conditioners ${ }^{(15)(16)}$, other than our teams' previous reports, there have been no studies of the predictive adjustment of the tradeoff between RTP and comfort made in intervals of minutes.

In our previous research, we defined an evaluation function that expressed the tradeoff between electricity charges and room-temperature comfort, and we used a simulated annealing (SA) algorithm to search for an power limitation command value permutation where this function was at its minimum ${ }^{(17)-(19)}$. However, although several multi-unit building air conditioners are normally installed in a single building, the evaluation function defined in our previous studies adjusted air conditioners individually, so the power adjusted for the building as a whole and the room temperature could not be controlled. For example, if the maximum allowed consumed power for an entire building is set by a contract with the electric company, the allowed value might be exceeded if consumed power were concentrated in low electricity charge unit slots. Also, conventional methods did not distinguish the room temperature comfort of each multi-unit building air conditioner, so it was not possible to take a lead in controlling the tradeoff between electricity charges and comfort among air conditioners that place a high priority on comfort and those that placed a low priority on comfort.

The present study defines a complex evaluation function 
that integrates and controls the electric power and room temperature comfort of multi-unit building air conditioners throughout a building. This evaluation function establishes a penalty for exceeding the allowed electric power quantity of the entire building and a function for room temperature priority, thereby allowing give-and-take adjustment of the power consumption between air conditioners. In order to verify the results of the complex evaluation function, complex RTP adjustment control was performed for an office building where multi-unit building air conditioners were in operation. However, the opportunity to perform tests with actual equipment is limited, and reproducibility is difficult to ensure. Therefore, we developed a mathematical formula model Aircon Emulator that imitated the power and room temperature changes of a multi-unit building air conditioner.

\section{Real-time Electricity Charges and Multi-unit Building Air Conditioners}

2.1 Real-time Electricity Charges Figure 1 shows a concept diagram of an RTP electricity charge adjustment control system for the multi-unit building air conditioner envisioned in this study. In the envisioned system, an aggregator receives a real-time charge notification from the electric company and then notifies the building energy management system (BEMS) of a power limitation command that is appropriate considering future electric charges. BEMS orders the power limitation command $P_{L_{m}}^{b}[\mathrm{~kW}]\left(P_{L_{m}}^{1}, P_{L_{m}}^{2}, \ldots\right)$ to each outdoor unit of the multi-unit building air conditioner to control the power $P^{b}(t)[\mathrm{kW}]\left(P^{1}(t), P^{2}(t), \ldots\right)$, with the aim of adjusting a tradeoff over the entire building that ensures the allowed power quantity for the full building and the room temperature comfort of the priority block. Here, the superscript $b$ is the air conditioner number $\left(b=1,2, \ldots, b_{\max }\right)$, the subscript $m$ is the control frame number every 5 minutes ( $m$ $=1,2, \ldots, M)$, and $t$ is the discrete time for each minute.

Figure 2 shows the relationship between the time slot $s$ of the real-time electricity charge envisioned in this study and the control frame $m$ of the power limitation command. $P_{L_{1}}^{b}, \ldots, P_{L_{m}}^{b}$ is the power limitation command of air conditioner $b$. The electricity charge is envisioned to change greatly for each time slot, where the time range $\tau_{\mathrm{s}}$ is $10 \mathrm{~min}$ utes. The aggregator uses the adjustment algorithm to calculate the power limitation command in the control frame range $\tau_{\mathrm{m}}$, which is shorter than the electricity charge time slot. At this time, the electricity charge $R_{\mathrm{S}}$ that was notified once is presumed not to be changed within the corresponding charge time slot.

2.2 Complex Adjustment of a Multi-unit Building Air Conditioner Group In the multi-unit building air conditioner, several indoor units are connected to a single outdoor unit via refrigerant piping. In a multi-unit building air conditioner, adjustment control of the flow rate of the complex system of refrigerant pipes and protection control for compression lubricant oil management are always being performed, so the actual time series data of power consumption and room temperature is complicated and ever fluctuating. Also, medium to large size office buildings normally have several multi-unit building air conditioners. In the present study, the integration of power consumption and room temperature is controlled for the whole building by providing a

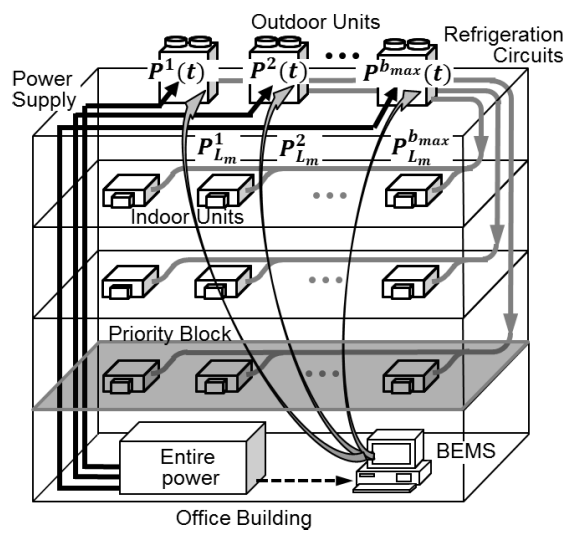

Fig. 1. Schematic diagram of real-time pricing optimization control for office building multi-type airconditioning facilities

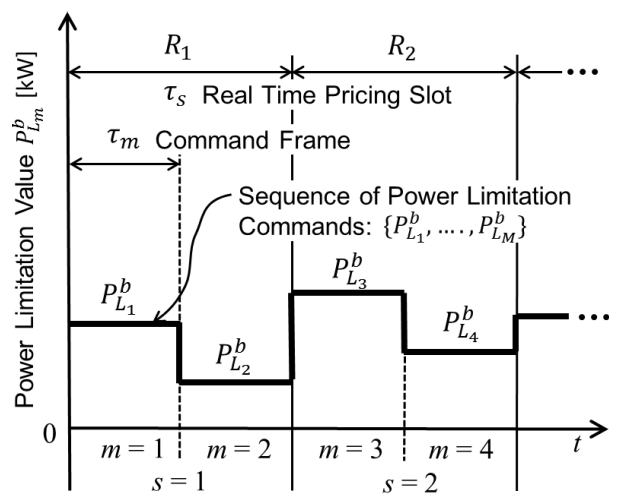

Fig. 2. Definition of smart grid real-time pricing time slots and power limitation command time frames

power limitation command value to a plurality of multi-unit building air conditioners. In addition, we prepare a neural network model for each multi-unit building air conditioner in order to predict the power consumption and room temperature response required to achieve such control, and we use this model to calculate the adjustment algorithm.

\section{Creating a Formula for the Problem of RTP Adjustment}

3.1 Conventional Simple RTP Adjustment Control

The present RTP adjustment problem uses an evaluation function that includes an index that balances economic performance and air conditioning comfort. The evaluation function for a single multi-unit building air conditioner is defined by the following formula, using the balance adjustment coefficient of economic performance and air conditioning comfort $\alpha$.

$$
E_{I}^{b}=\alpha \sum_{s=1}^{S_{\max }} R_{s} \cdot W_{s}^{b}+(1-\alpha) \sum_{s=1}^{S_{\max }} Z_{s}^{b} \cdots \cdots \cdots \cdots
$$

Here, $b$ is the number of the multi-unit building air conditioner $\left(b=1,2, \ldots, b_{\max }\right), s$ is the charge time slot number $(s$ $\left.=1,2, \ldots, S_{\text {max }}\right), R_{\mathrm{S}}$ is the charge unit price $[\mathrm{JPY} / \mathrm{kWh}], W_{s}^{b}$ is the power consumption quantity [kWh], and $Z_{s}^{b}$ is the room temperature penalty $\left[\mathrm{deg}^{2}\right] . W_{s}^{b}$ is integrated by the following formula. 


$$
W_{s}^{b}=\frac{1}{60} \sum_{m=1}^{m_{\max }} \sum_{t^{\prime}=1}^{t_{\max }^{\prime}} \tilde{P}^{b}\left(s \cdot \tau_{s}+m \cdot \tau_{m}+t^{\prime}\right) \ldots \ldots \ldots
$$

$m$ is the control frame number $\left(m=1,2, \ldots, m_{\max }\right), \tau_{\mathrm{s}}$ is the charge time frame length, and $\tau_{\mathrm{m}}$ is the control frame length, and if $t^{\prime}$ is the 1-minute interval time within the control frame length, then $W_{s}^{b}$ is the integration of a single charge time slot portion of the power consumption prediction value $\tilde{P}^{b}\left(s \cdot \tau_{\mathrm{s}}+m \cdot \tau_{\mathrm{m}}+t^{\prime}\right)$ for each minute. The reason for dividing by 60 is to obtain $\mathrm{kWh}$ as the units of the power consumption for each minute. Also, $Z_{s}^{b}$ is integrated by the following formula.

$$
\begin{aligned}
& Z_{s}^{b}=\sum_{m=1}^{m_{\max }}\left(\tilde{T}_{S A}^{b}\left(s \cdot \tau_{s}+m \cdot \tau_{m}+\tau_{m}-1\right)\right)^{2} \ldots \ldots \\
& T_{S A}^{b}(t)=\left\{\sum_{i=1}^{N_{I U}^{b}} C_{i}^{b} \cdot\left(T_{A i}^{b}(t)-T_{S i}^{b}(t)\right)\right\} / \sum_{i=1}^{N_{I U}^{b}} C_{i}^{b} \ldots
\end{aligned}
$$

Here, $T_{S A}^{b}(t)$ is the averaged room temperature deviation, which is used as one index of air conditioning comfort in a single multi-unit building air conditioner. $i$ is the indoor unit number $\left(i=1,2, \ldots, N_{I U}\right), T_{A i}^{b}(t)$ is the room temperature sensor value of each indoor unit, $T_{S i}^{b}(t)$ is the set temperature of each indoor unit, $C_{i}^{b}$ is the rated cooling capacity of an indoor unit, and $N_{I U}^{b}$ is the number of indoor units of air conditioner $b$. Smaller values of $T_{S A}^{b}(t)$ mean higher values of comfort in the rooms where air conditioning is controlled by the multi-unit building air conditioner.

Here, the permutation of the power limitation command $P_{L_{m}}^{b}$ up to the control frame number $(m=1,2, \ldots, M)$ is expressed by the following formula.

$$
\left\{P_{L_{m}}^{b}\right\}_{m=1}^{M}=\left\{P_{L_{1}}^{b}, P_{L_{2}}^{b}, \ldots, P_{L_{M}}^{b}\right\}
$$

With the adjustment methods described in previous studies, in the permutation space of $M=m_{\max } \times S_{\text {max }}$ dimensionality, we had been trying to find a permutation of the power limitation command value that achieves the minimum evaluation function $E_{I}^{b}\left(\left\{P_{L_{m}}^{b}\right\}_{m=1}^{M}\right)$ in which formula (1) is substituted into formula (5). The following formula is an evaluation function that summarizes formula (1) with regard to a whole building air conditioner.

$$
E_{I}=\sum_{b=1}^{b_{\max }} E_{I}^{b}\left(\left\{P_{L_{m}}^{b}\right\}_{m=1}^{M}\right) \ldots \ldots \ldots \ldots \ldots \ldots \ldots \ldots
$$

The minimum value of the above formula is the total value of the minimum values of each multi-unit building air conditioner. In other words, the state of an individual multi-unit building air conditioner does not affect the adjustment results of other multi-unit building air conditioners.

3.2 Evaluation Function of Complex RTP Adjustment Control When there is an urgent demand for power, the power consumption of the entire building must be reduced in order to not exceed the contract power consumption with the electric company. At this time, specific multi-unit building air conditioners installed in reception rooms and server rooms, for example, should be operated in a way that does not negatively affect room temperature comfort. Thus, we changed the evaluation function of formula (1) to generate a penalty for exceeding the allowed value of the 5-minutes power consumption of the entire building. The room temperature penalty is also provided with a priority level for each multi-unit building air conditioner. The evaluation function is as follows.

$$
\begin{aligned}
& E_{C}=\alpha \sum_{s=1}^{S_{\max }} \sum_{b=1}^{b_{\max }} R_{s} W_{s}^{b}+(1-\alpha) \sum_{s=1}^{S_{\max }} \sum_{b=1}^{b_{\max }} Z_{C s}^{b}+\sum_{s=1}^{S_{\max }} X_{s}^{\text {all }} \\
& \sum_{b=1}^{b_{\max }} \beta^{b}=0 \\
& \text {.............. (7) } \\
& Z_{C s}^{b}=\left[\left(1+\beta^{b}\right) \tilde{T}_{S A}^{b}\left(s \cdot \tau_{s}+m \cdot \tau_{m}+\tau_{m}-1\right)\right]^{2} \\
& X_{s}^{\text {all }}=\left\{\begin{array}{l}
\gamma\left[\sum_{b=1}^{b_{\max }} W_{s}^{b}-W_{5 \max }^{\text {all }}\right]^{2} \\
\ldots\left(\text { if } \sum_{b=1}^{b_{\max }} W_{s}^{b}>W_{5 \max }^{\text {all }}\right) \\
\ldots(\text { otherwise })
\end{array}\right.
\end{aligned}
$$

Here, $Z_{C s}^{b}$ is the priority room temperature penalty $\left[\mathrm{deg}^{2}\right], \beta^{\mathrm{b}}$ is the room temperature priority, $X_{s}^{\text {all }}$ is the penalty for exceeding the whole building peak powerconsumption, $W_{5 \max }^{\text {all }}$ is the full-building 5-minute allowed power consumption [kWh]. $\gamma$ is the power consumption exceeding penalty coefficient, the value with which the size of $X_{s}^{\text {all }}$ is adjusted. Adjustment by formula (7) controls the power consumption of each air conditioner so that the penalty for exceeding the whole building power consumption $X_{s}^{\text {all }}$ is generated as little as possible and $\tilde{T}_{S A}^{b}$ with high room temperature priority $\beta^{\mathrm{b}}$ is as small as possible. In other words, the power limitation command value of an air conditioner for the whole building is the search target of the adjustment algorithm, and its permutation is as follows.

$$
\left\{\left\{P_{L_{m}}^{b}\right\}_{m=1}^{M}\right\}_{b=1}^{b_{\max }}=\left\{\left\{P_{L_{\mathrm{m}}}^{1}\right\}_{m=1}^{M},\left\{P_{L_{m}}^{2}\right\}_{m=1}^{M}, \ldots,\left\{P_{L_{m}}^{b_{\max }}\right\}_{m=1}^{M}\right\}
$$

To address the problem of optimizing this formulation, (a) the decision variable is formula (11), which is the permutation of the power limit $P_{L_{m}}^{b}$; (b) the target functions for optimization are formulas (7) to (10); and (c) the constraint conditions are $P_{L_{m}}^{b}=P_{\text {max }}^{b} \cdot n_{L} /\left(N_{L}-1\right)$. Here, $N_{L}$ is the number of steps of discretization, $P_{\text {max }}^{b}$ is the rated power consumption $[\mathrm{kW}], n_{L}$ is the command step $\left(n_{L}=0,1, \ldots, N_{L}-1\right)$, and $P_{L_{m}}^{b}$ is confined to the range $0 \leq P_{L_{m}}^{b} \leq P_{\text {max }}^{b}$.

\section{RTP Adjustment Search Algorithm}

4.1 Simulated Annealing Algorithm In this report, we extract only the difference between simple control and complex control under the same algorithm method conditions as that of previous research ${ }^{(17)-(19)}$. Therefore, similar to previous research, the search algorithm of the adjustment solution is Simulated Annealing ${ }^{(20)(21)}$, and a permutation of the power limitation command value that achieves the minimum value $E_{c}\left(\left\{\left\{P_{L_{m}}^{b}\right\}_{m=1}^{M}\right\}_{b=1}^{b_{\max }}\right)$ of formula (11) substituted into formula (7) in permutation space of $b_{\text {max }} \times m_{\text {max }} \times S_{\text {max }}$ dimensionality. 
As far as the reason for applying SA in previous research, a model of the interplay between air conditioning power and room temperature was made using a neural network and the evaluation function was multimodal, so SA was selected as an example of a random search method.

SA is believed to require a long calculation time. However, with the target of the present study, when $N_{L}=5, b_{\max }=5$, and $M=2$, approximately 1000 times, and even on a normal $\mathrm{PC}$, the allowed convergence range is reached in 1 second or less, so there is no problem with a control frequency of $\tau_{\mathrm{m}}=$ 5 min.

4.2 Algorithm Execution Conditions The search algorithm conditions were set as follows. (a) A random value was used for the initial state of the decision variable $P_{L_{m}}^{b}$, and $P_{L_{m}}^{b}=P_{\max }^{b} \cdot R /\left(N_{L}-1\right)$. Here, $R$ is a uniform random number $\left(R=0,1, \ldots, N_{L}-1\right)$. (b) Toward the adjacent state, 1 part of the permutation of $P_{L_{m}}^{b}$ is changed, i.e., a single air conditioner $b$ and control frame $m$ are selected based on a uniform random number, and $P_{L_{m}}^{b}$ is changed with the same formula as in (a). (c) The temperature is reduced with $T_{\text {Anneal }}$ $=k_{r} \exp \left(-i_{S A} / i_{S A \max }\right)$. Here, $i_{S A}$ is the search count, $i_{S A \max }$ is the maximum search count, and $k_{r}$ is the reduction parameter. Even when searching was performed according to the above formula, it was performed in such a way as to maintain the random transition until the end, without lowering the temperature to near 0 .

With SA, the solution may differ according to the initial value and the random number, so we evaluated the statistical difference between the simple control and complex control by repeating the simulation with different initial values and random numbers.

4.3 Power/room Temperature Response Prediction Model This study uses a 4-layer perceptron neural network in a model that predicts the future power consumption and room temperature when a power limitation command is given. According to findings obtained from previous studies ${ }^{(22)-(28)}$, the input variable $\boldsymbol{x}(t)$ and the output variable $\boldsymbol{y}(t)$ are as follows.

$$
\begin{aligned}
& \boldsymbol{x}(t)= {\left[P_{L}^{b}(t+1), P_{L}^{b}(t), P^{b}(t-4), \ldots, P^{b}(t-1), P^{b}(t)\right.} \\
&\left.T_{S A}^{b}(t-4), \ldots, T_{S A}^{b}(t-1), T_{S A}^{b}(t), T_{O}^{b}(t)\right]^{T} \\
& \ldots \ldots \ldots \ldots \ldots \ldots(12) \\
& \boldsymbol{y}(t)= {\left[\tilde{P}^{b}(t+1), \ldots, \tilde{P}^{b}(t+5),\right.} \\
&\left.\tilde{T}_{S A}^{b}(t+1), \ldots, \tilde{T}_{S A}^{b}(t+5)\right]^{T} \ldots \ldots \ldots(13)
\end{aligned}
$$

The relationship between the input variable and the output variable is described by the following formula.

$$
y_{l}(\boldsymbol{x})=\sum_{k=1}^{K} w_{k l} \times \operatorname{Re}\left(\sum_{j=1}^{J} v_{j k} \times \operatorname{Re}\left(\sum_{i=1}^{I} u_{i j} \times \boldsymbol{x}_{i}\right)\right)
$$

Here, $T_{O}^{b}(t)$ is the outer air temperature $\left[{ }^{\circ} \mathrm{C}\right], \operatorname{Re}(\cdot)$ is the Rectified Linear Unit function, and $u_{i j}, v_{j k}, w_{k l}$ are the weight coefficients that link between NN elements. $I=13$ is the input layer element number, $J=12$ is the 1 st hidden layer element number, $K=11$ is the 2 nd hidden layer element number, and $L=10$ is the output layer element number.

\section{Dynamic Characteristic Model of a Multi-unit Building Air Conditioner}

In order to verify the adjustment algorithm, we developed Aircon Emulator (AE), a multi-unit building air conditioner real-time dynamic characteristics model. This model imitates the power consumption of a multi-unit building air conditioner and the temperature changes in each indoor unit. The fundamental equation is based on the traditional heat balance equation ${ }^{(29)}$ that depends on heat capacity, heat transmittance, and internal heat generation. On the air conditioning side, a model was made of independent room temperature control of each indoor unit and acceleration/deceleration control of the compressor inverter.

The characteristic of this model is that it includes a thermooff function that stops cooling when the measured room temperature of each indoor unit approaches the set temperature. The changes of power consumption and room temperature calculated by the AE model depend on the thermo-off/on status of each indoor unit.

A mathematical formula of the model is as follows.

$$
\begin{aligned}
& P^{b}(t+1)=P^{b}(t)+D^{b}(t) \\
& D^{b}(t)=S_{F R}^{b}\left\{S_{T H O}^{b} D_{U P}^{b}+\left(1-S_{T H O}^{b}\right) D_{D W N}^{b}\right\} \\
& +\left(1-S_{F R}^{b}\right) S_{O V}^{b} D_{D W N}^{b} \\
& +\left(1-S_{O V}^{b}\right)\left(S_{T H O}^{b} D_{H L D}^{b}+\left(1-S_{T H O}^{b}\right) D_{D W N}^{b}\right) \\
& \text {............... (16) } \\
& T_{A i}^{b}(t+1)=T_{A i}^{b}(t)-k_{A i}^{b} \eta_{C O P}^{b} \frac{C_{P i}^{b}}{\sum_{i \in b}\left(C_{P i}^{b} \cdot S_{T H i}^{b}\right)} P^{b}\left(t-t_{D i}^{b}\right) \\
& +k_{O}^{b}\left(T_{O}^{b}(t)-T_{A i}^{b}(t)\right)+k_{I}^{b} P_{I i}^{b} \\
& T_{A}^{b}(t+1)=\sum_{i=1}^{N_{I U}^{b}}\left(C_{P i}^{b} \cdot T_{A i}^{b}(t+1)\right) / \sum_{i=1}^{N_{I U}^{b}} C_{P i}^{b} \cdots \cdots
\end{aligned}
$$

\begin{tabular}{|c|c|}
\hline Symbol & Parameter \\
\hline$D_{U P}^{b}$ & Power up rate $[\mathrm{kW} / 10 \mathrm{~s}]$ \\
\hline$D_{D W N}^{b}$ & Power down rate $[\mathrm{kW} / 10 \mathrm{~s}]$ \\
\hline$D_{H L D}^{b}$ & Holding power of the time $[\mathrm{kW} / 10 \mathrm{~s}]$ \\
\hline$\eta^{b}{ }_{C O P}$ & Coefficient Of Performance (COP) of aircon $b[-]$ \\
\hline$C_{P i}^{b}$ & Cooling capacity of indoor unit $i[\mathrm{~kW}]$ \\
\hline$k_{A i}^{b}$ & Coefficient of cooling power $\left[{ }^{\circ} \mathrm{C} / \mathrm{kW}\right]$ \\
\hline$k_{O}^{b}$ & Coefficient of heat transmission from outside [-] \\
\hline$k_{I}^{b}$ & Coefficient of internal heat load $\left[{ }^{\circ} \mathrm{C} / \mathrm{kW}\right]$ \\
\hline$P_{I i}^{b}$ & Internal heat load of indoor unit $i[\mathrm{~kW}]$ \\
\hline$t_{D i}^{b}$ & Cooling delay $[10 \mathrm{~s}]$ \\
\hline
\end{tabular}

Here, $t$ is the discrete time for every 10 seconds, $P^{b}(t)$ is the power consumption [kW] of air conditioner $b, D^{b}(t)$ is the power change [kW] every 10 seconds, $T_{A i}^{b}(t)$ is the room temperature $\left[{ }^{\circ} \mathrm{C}\right]$ of indoor unit $i$, and $T_{O}^{b}(t)$ is the outside air temperature $\left[{ }^{\circ} \mathrm{C}\right]$. Also, the room temperature of each indoor unit weight-averaged with rated cooling capacity is defined as $T_{A}^{b}(t)$, and it is used as the averaged room temperature of air conditioner $b$. Table 1 lists the constants included in the above formula. $S_{F R}^{b}$ and $S_{O V}^{b}$ are the tracking states of power

Table 1. AE parameter description 
Complex Coordination Control with Entire Power Limitation and Partial Temperature Priority（Yoshifumi Aoki et al.)

Table 2. Conditions of AE control status

\begin{tabular}{c|c}
\hline Valiable & \multicolumn{1}{|c}{ Conditions and values } \\
\hline$S_{F R}^{b}$ & if $P^{b}(t)<k_{P_{L}}^{b} \cdot P_{L}^{b}(t)$ then 1, otherwise 0 \\
\hline$S_{T H O}^{b}$ & if $\sum_{i \in b} S_{T H i}^{b}>0$ then 1, otherwise 0 \\
\hline$S_{O V}^{b}$ & if $P^{b}(t)>k_{P_{I}}^{b} \cdot P_{L}^{b}(t)$ then 1, otherwise 0 \\
\hline$S_{T H i}^{b}$ & $\begin{array}{l}\left.\text { if } S_{T H i}^{b}=0 \text { (if } T_{A i}^{b}(t)-T_{S i}^{b}(t) \leq \delta T_{T H O F F}^{b} \text { then } S_{T H i}^{b}=1\right) \\
\left.\text { otherwise (if } T_{A i}^{b}(t)-T_{S i}^{b}(t) \geq \delta T_{T H O N}^{b} \text { then } S_{T H i}^{b}=0\right)\end{array}$ \\
\hline
\end{tabular}

$P^{b}(\mathrm{t})$ with respect to power limitation command value $P_{L}^{b}(t)$, $S_{T H O}^{b}$ is the operation state of the external unit, and $S_{T H i}^{b}$ is the variable that expresses the thermo-off/on state of an indoor unit. These values change under the conditions shown in Table 2. In Table $2, k_{P_{L}}^{b}\left(0<k_{P_{L}}^{b} \leq 1\right)$ is the $P^{b}$ tracking ratio to the magnitude of $P_{L}^{b}(t)$, and it changes according to the magnitude of $P_{L}^{b}(t) . \delta T_{T H O F F}^{b}$ and $\delta T_{T H O N}^{b}$ are the hysteresis during thermo-off/on.

\section{Verification of the AE Model with an Actual Equipment Test}

6.1 Actual Equipment Test Conditions A summary of the office building and multi-unit building air conditioner used in the actual equipment test is shown in Table 3.

The test was performed during a 30 minute period from 12:30 to 13:00 on a weekday in August 2017. Operation of the air conditioner was shut down for lunch break starting at 12:00, and the room temperature at the start of the test was approximately $30^{\circ} \mathrm{C}$. The condition for this test was a marked tradeoff between the restoration of room temperature comfort and massive power consumption, which experts call "pulldown", and it was the condition setting on which our research focused.

The set temperature of each indoor unit $T_{S i}^{b}$ was $26\left[{ }^{\circ} \mathrm{C}\right]$. For the evaluation of electricity charges, the three stages of high amount $R_{H}=100[\mathrm{JPY} / \mathrm{kWh}]$, low amount $R_{L}=$ 10 [JPY $/ \mathrm{kWh}$, and moderate amount $R_{M}=30[\mathrm{JPY} / \mathrm{kWh}]$ were established, and the unit price pattern changed in the or$\operatorname{der}\left\{R_{1}, R_{2}, R_{3}\right\}=\left\{R_{H}, R_{L}, R_{M}\right\}$ at 10 minute intervals. Also, as an example, the evaluation function parameters for both simple and complex adjustment evaluation functions were $\alpha$ $=0.1$. The parameters of the complex evaluation function were $\beta^{b}=\{0.5,0.0,-0.5,-0.5,0.5\}$ (in order of $b=1$ to 5 ), $\gamma=10$, and $W_{5 \max }^{\text {all }}=2.33[\mathrm{kWh}]$. Here, $W_{5 \max }^{\text {all }}$ was a value equal to or greater than the total value of the lowest power consumption $P_{\text {min }}^{b}[\mathrm{~kW}]$ of each air conditioner that allowed confirmation of the adjustment control effect, and it was calculated by the following formula.

$$
W_{5 \max }^{\text {all }}=2 \times \frac{5}{60} \sum_{b=1}^{b_{\max }} P_{\min }^{b}
$$

\subsection{Comparison of the Test Results and Simulation}

In this instance, for simple RTP adjustment control and complex RTP adjustment control, only 1 test each was performed with regard to 1 unit price pattern, and the control results are only one example of stochastic behavior. Therefore, with simulation using $\mathrm{AE}$, we performed a comparison when the unit price pattern permutation was changed.

In order for the behavior to match the general response,
Table 3. Outline of a sample facility

\begin{tabular}{l|l}
\hline \multicolumn{1}{c|}{ Item } & \multicolumn{1}{c}{ Specification } \\
\hline Type of building & General purpose office \\
\hline Dimension & 2 stories, area app. $1600 \mathrm{~m}^{2}$ \\
\hline No. of outdoor units & 5 outdoor units \\
\hline No. of indoor units & 6 for each outdoor unit, total 30 \\
\hline Rated cooling capacity & $45 \mathrm{~kW}, 40 \mathrm{~kW}, 68 \mathrm{~kW}, 73 \mathrm{~kW}, 45 \mathrm{~kW}$ \\
\hline
\end{tabular}

the same power limitation command $P_{L_{m}}^{b}$ as in the experiment was entered variously, and parameters were adjusted so that $\mathrm{AE}$ was equivalent to the actual equipment. The operations in the actual equipment of Simple-RTP are shown in Fig. 3, and the results of the AE simulation are shown in Fig. 4. Also, the control results in the Complex-RTP are shown in Fig. 5, and the simulation results are shown in Fig. 6.

A permutation of the same power limitation command as that during each actual equipment test was given to $\mathrm{AE}$ and operated. Regarding power, the error between the actual equipment and $\mathrm{AE}$ was a mean of $8.5 \%$ in the rated power ratio, and the error in the room temperature was $0.5\left[{ }^{\circ} \mathrm{C}\right]$ on average. The primary cause of the power error was the instrument protection control circled in Fig. 3 and Fig. 5. This instance was not the target of the simulation.

Graphs summarizing the power, room temperature, and whole building 5-minute power consumption of each air conditioner in the actual equipment are shown in Fig. 7. Also, graphs similarly summarizing this information for $\mathrm{AE}$ are shown in Fig. 8. In the graph of $W_{5}^{\text {all }}$ showing the whole building 5-minute power consumption of Simple-RTP, $W_{5 \max }^{\text {all }}$ shown by the dotted line is greatly exceeded in frames $R_{2}$ and $R_{3}$. This is because in the simple evaluation function defined in formula (1), the power consumption and room temperature are adjusted without regard to other air conditioners, so it is not possible to prevent the allowed power from being exceeded. Meanwhile, in the graph of $W_{5}^{\text {all }}$ of the Complex-RTP shown in Fig. 7(b), it stops near $W_{5 \max }^{\text {all }}$ in all price frames. In the upper row $P^{b}$ graph showing the power of each air conditioner, power is alternately regulated to allow a give and take in power consumption between each of the air conditioners, especially during the interval from 15 to 30 minutes. Regarding the effect of the power limitation command on room temperature comfort, the room temperature in which the room temperature priority $\beta^{\mathrm{b}}$ is prioritized (Block $b=1$, Block $b$ $=5$ ) rapidly approaches the set temperature of $26\left[{ }^{\circ} \mathrm{C}\right]$, but 2 of the non-prioritized room temperatures (Block $b=3$, Block $b=4$ ) are controlled so that they change gradually. The AE simulation results shown in Fig. 8 also show a similar trend to that of the actual equipment test that was described above.

$\mathrm{SA}$ is a stochastic search method, so in the following section, 100 simulations are performed, and the control results are statistically evaluated.

\section{Effect of Whole Building Complex RTP Ad- justment Control}

In order to verify the efficacy of complex RTP adjustment control, AE was used to perform a simulation in which the unit price pattern was changed in various ways. The conditions of the simulation were pulldown, similar to the actual equipment test, and operation was simulated for a 30 minute 


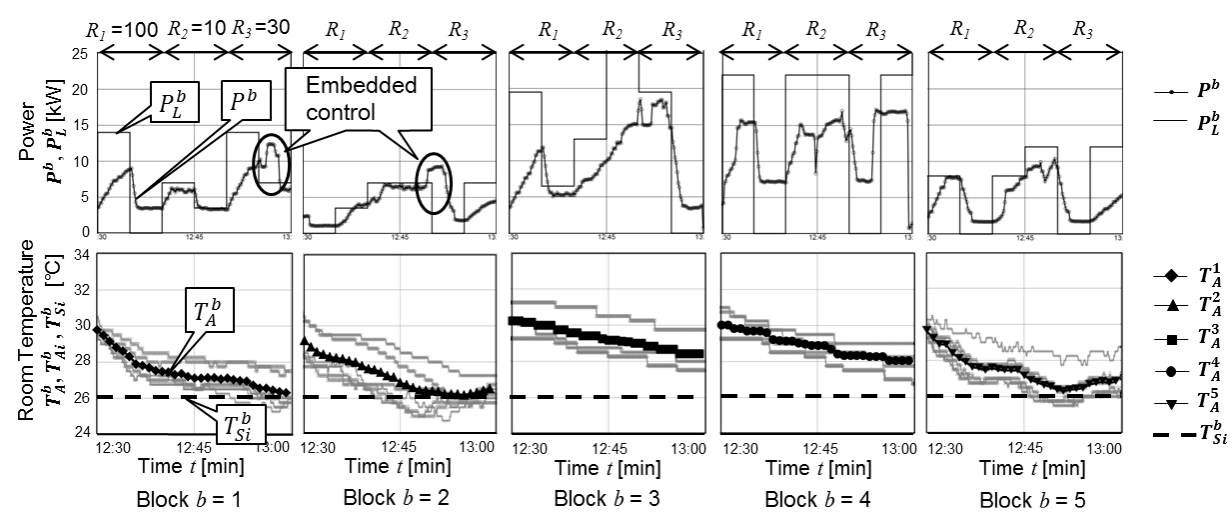

Fig. 3. Experiment of Simple-RTP optimization using the actual air-conditioning facilities
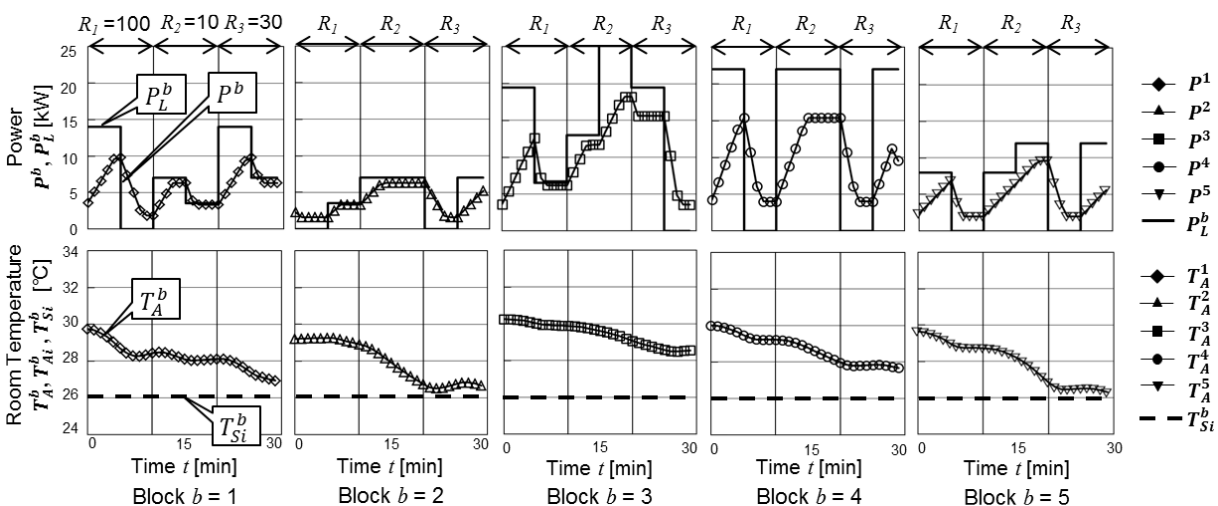

Fig. 4. Simulation of Simple-RTP optimization using AE models with the identical $P_{L}$ permutation

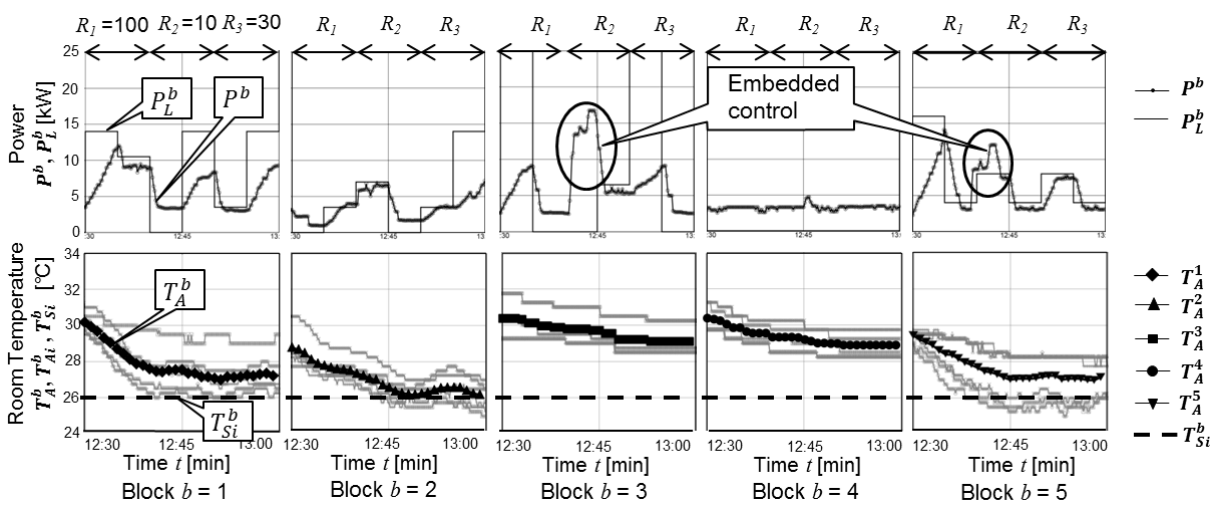

Fig. 5. Experiment of Complex-RTP optimization using the actual air-conditioning facilities

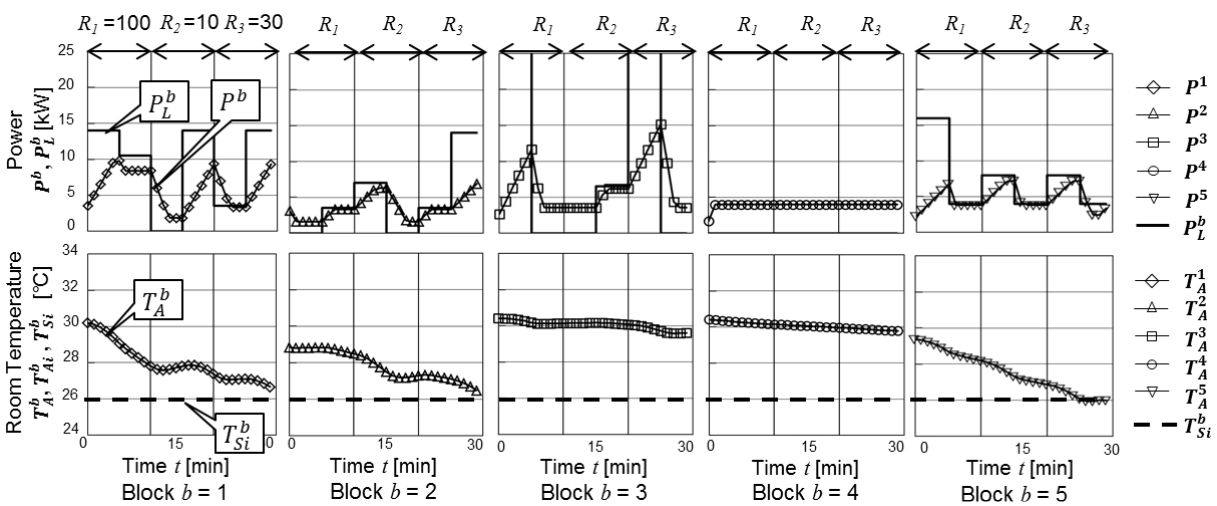

Fig. 6. Simulation of Complex-RTP optimization using AE models with the identical $P_{L}$ permutation

period starting at an initial temperature of $30\left[{ }^{\circ} \mathrm{C}\right]$. In order to focus on results according to differences in the unit price pattern, the AE was used to create a model that corresponded to
Block $b=1$ throughout, and evaluation function parameters $\alpha, \beta^{\mathrm{b}}$, and $\gamma$ were the same values as in the actual equipment test. $W_{5 \max }^{\text {all }}$ was calculated according to formula (19) to be 


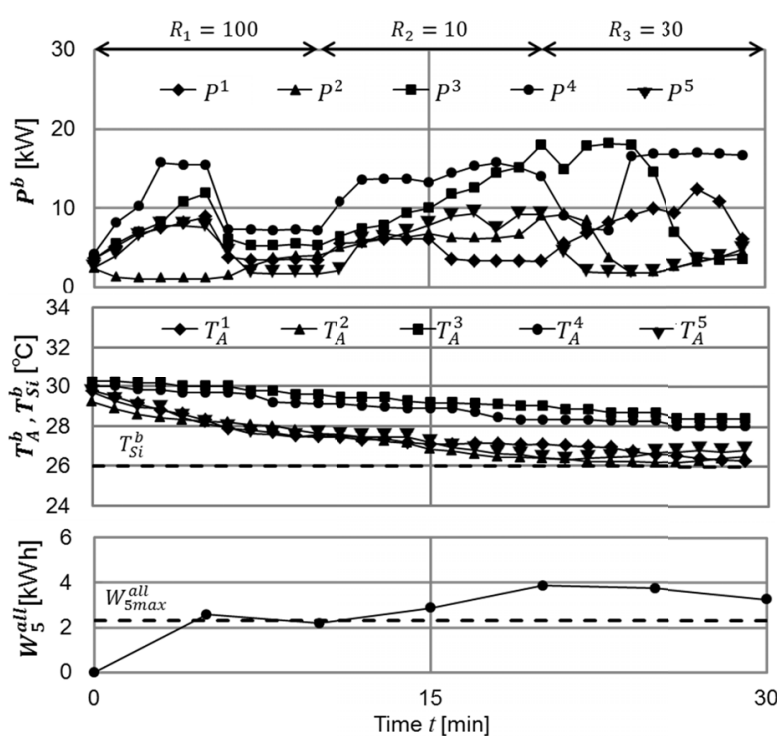

(a) Simple-RTP

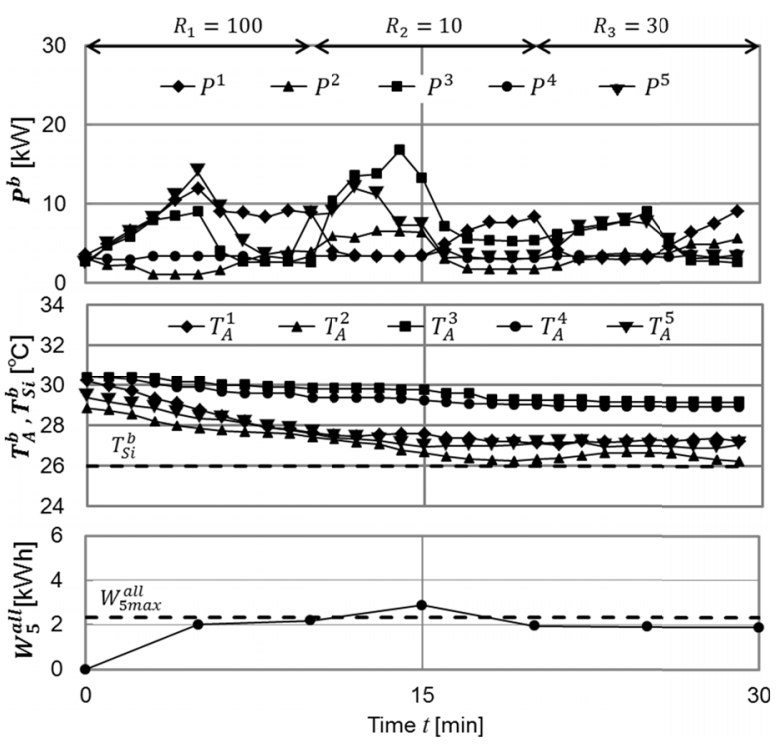

(b) Complex-RTP

Fig. 7. Experiment results of Simple-RTP and Complex-RTP optimization

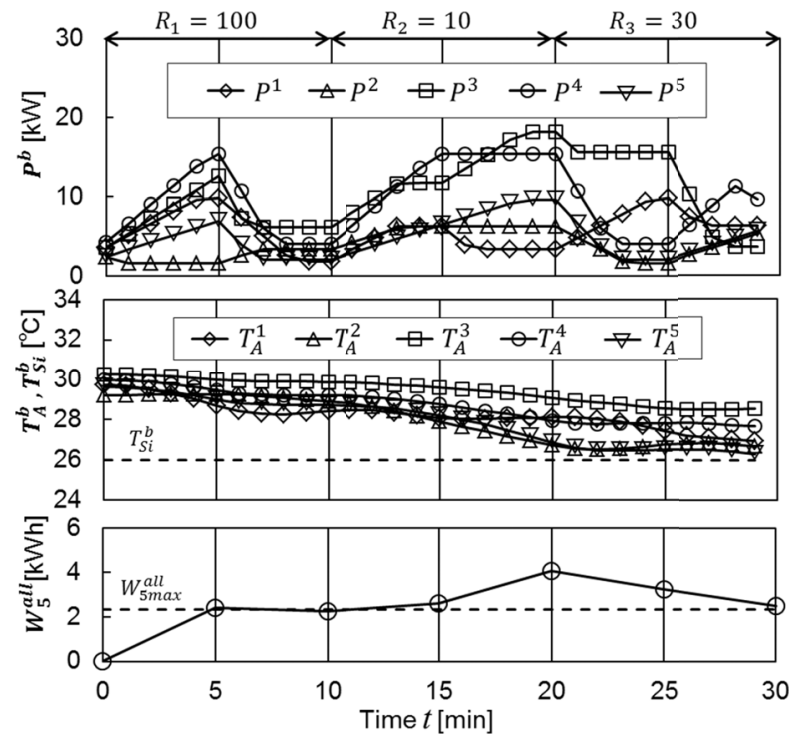

(a) Simple-RTP

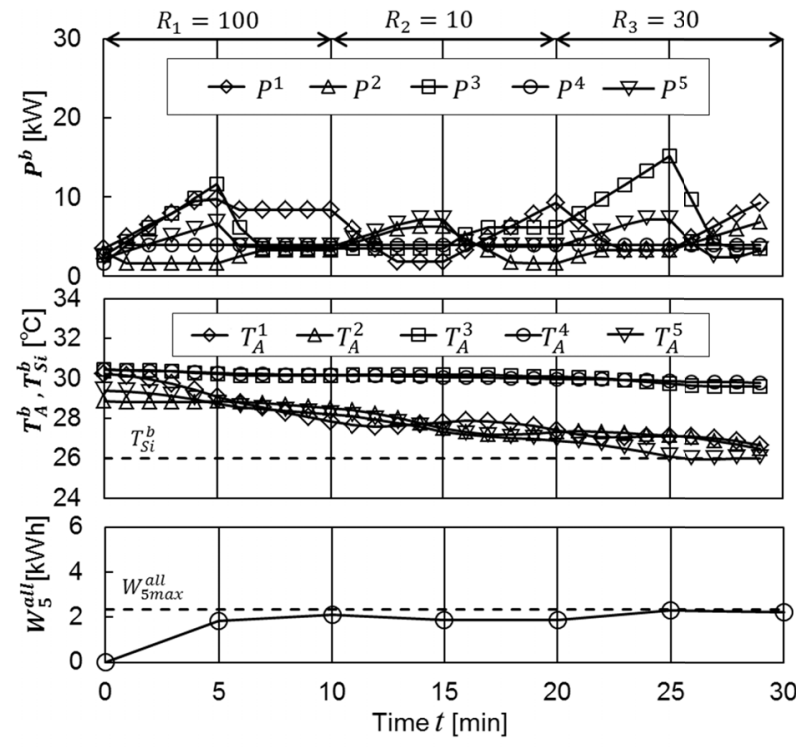

(b) Complex-RTP

Fig. 8. Simulation results of Simple-RTP and Complex-RTP optimization

$1.53[\mathrm{kWh}]$. Regarding all control frames, for each or normal operation (Normal) in which $P_{L_{m}}^{b}$ is not applied, Simple-RTP, and Complex-RTP, a simulation was performed with a $3 !=6$ way unit price pattern in order to cover all of the permutations of $R_{H}, R_{L}$, and $R_{M}$.

For each unit price pattern, the 30-minute whole building total power consumption $W_{30}^{\text {all }}$, whole building electricity charge $Y_{30}^{\text {all }}$, and the 30-minute composite average room temperature deviation $T_{A D}^{p r i}$ of the air conditioner in which room temperature priority was set with priority $\left(\beta^{\mathrm{b}}=0.5\right)$ are summarized in Figs. 9 to 11.

Figure 9 to Figure 11 show the mean value and $\pm 3 \sigma(\sigma$ $=$ standard deviation) distribution width (dotted line) when 100 simulations were performed. The distribution width is not written for Simple-RTP because $\sigma=0$. The $\pm 3 \sigma$ value of Complex-RTP is $8 \%$ or less of the mean value, so it is discussed as the mean value from here on out. Figure 9 shows

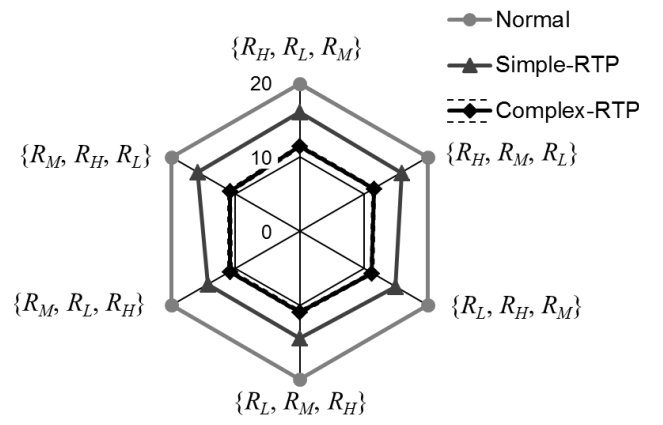

Fig. 9. Total power consumption $W_{30}{ }^{\text {all }}[\mathrm{kWh}]$ with various price patterns

the simulation results of the whole building total power consumption $W_{30}^{\text {all }}$. The reason that it is shown with a radar chart is to extract the unit price pattern dependence. $W_{30}^{\text {all }}$ 


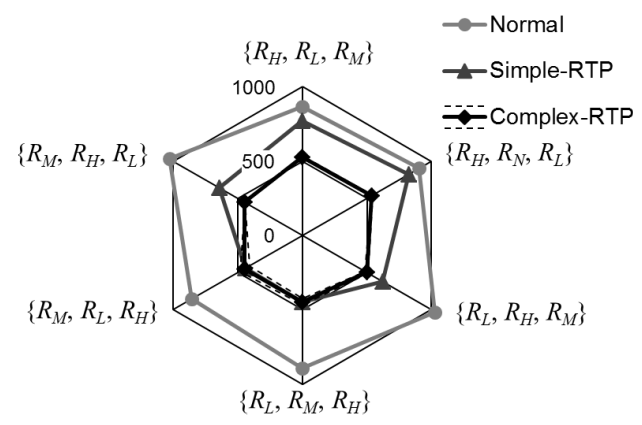

Fig. 10. Total electricity charge $Y_{30}$ all $[\mathrm{JPY}]$ with various price patterns

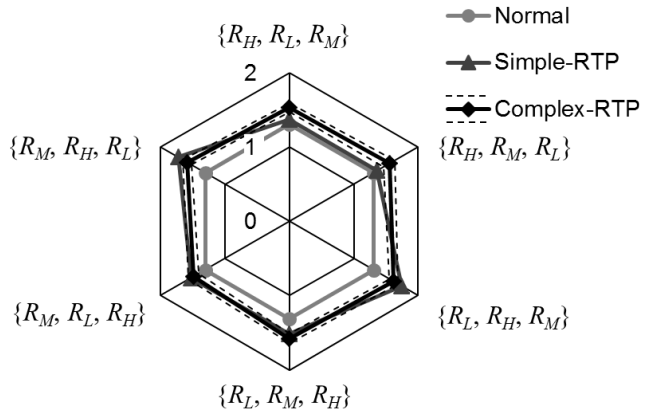

Fig. 11. Total average temperature deviation of priority areas $T_{A D}^{p r i}\left[{ }^{\circ} \mathrm{C}\right]$ with various price patterns

was $19.9[\mathrm{kWh}]$ and did not change in Normal and changed slightly from 14.3 to 16.1 [kWh] in Simple-RTP and from 10.8 to $11.5[\mathrm{kWh}]$ in Complex-RTP. $W_{30}^{\text {all }}$ decreased $27 \%$ in Complex-RTP, compared with Simple-RTP. Also, because it decreased "coaxially" regardless of unit price pattern, complex adjustment was shown to always have a larger power reduction effect than simple adjustment, the reason that $W_{30}^{\text {all }}$ of Normal does not change according to the unit price pattern is that power is not limited according to $P_{L_{m}}^{b}$.

Figure 10 shows a radar chart of the whole building total electricity charge $Y_{30}^{\text {all }}$. The range of $Y_{30}^{\text {all }}$ and the change rate of the minimum value/maximum value was 856 to 1030 [JPY] (17\% change) for Normal, 442-821 [JPY] (46\% change) for Simple-RTP, and 447 to 536 [JPY] (17\% change) for Complex-RTP. The change in $Y_{30}^{\text {all }}$ due to the unit price pattern was smaller for Complex-RTP than for Simple-RTP, and particularly in the so-called "falling unit price pattern" of $\left\{R_{H}, R_{L}, R_{M}\right\}$ and $\left\{R_{H}, R_{M}, R_{L}\right\}$ in which the initial frame was the maximum value, Complex-RTP cut electricity charges by $33 \%$ in comparison to Simple-RTP.

Figure 11 shows a radar chart of the total room temperature deviation $T_{A D}^{p r i}$ of the prioritized air conditioner. In Normal, it was $1.3\left[{ }^{\circ} \mathrm{C}\right]$ for all unit price patterns, in Simple-RTP, it was $1.4\left[{ }^{\circ} \mathrm{C}\right]$ to $1.7\left[{ }^{\circ} \mathrm{C}\right]$, and in Complex-RTP it was $1.5\left[{ }^{\circ} \mathrm{C}\right]$ to $1.6\left[{ }^{\circ} \mathrm{C}\right]$. The difference between Complex-RTP and SimpleRTP was largest in the $\left\{R_{H}, R_{L}, R_{M}\right\}$ and $\left\{R_{H}, R_{M}, R_{L}\right\}$ "falling unit price pattern", but the difference was only $+0.2\left[{ }^{\circ} \mathrm{C}\right]$.

\section{Discussion}

8.1 Whole Building Total Power Consumption/ Charge Complex effect The reason that the whole building total power consumption $W_{30}^{\text {all }}$ of the simulation in the previous section improved by $27 \%$ more than Simple-RTP was because the penalty $X_{s}^{\text {all }}$ for exceeding the power consumption limited the 5-minute power consumption to near $W_{5 \max }^{\text {all }}$. In this simulation, $W_{30}^{\text {all }}$ exceeded approximately 2.5 [kWh], but this excess was the tradeoff with $T_{A D}^{p r i}$, so it can be decreased by increasing $\gamma$. In other words, an overall adjustment, not just an adjustment for individual air conditioners, was shown to be possible in Complex-RTP by adjusting $W_{5 \max }^{\text {all }}$ and $\gamma$.

Compared with Simple-RTP, Complex RTP provided a $33 \%$ cost reduction effect in the whole building total electricity charge $Y_{30}^{\text {all }}$ in the $\left\{R_{H}, R_{L}, R_{M}\right\}$ and $\left\{R_{H}, R_{M}, R_{L}\right\}$ "falling unit price pattern." This was a marked difference. This difference stems from the fact that the operating conditions in this instance were pulldown from a room temperature of $30\left[{ }^{\circ} \mathrm{C}\right]$ and the initial room temperature penalty $Z_{s}^{b}$ was the maximum. In Simple-RTP, each air conditioner consumes power ignoring the whole building allowed power consumption, but in Complex-RTP, only the power of the prioritized air conditioner is consumed under the limitation $W_{5 \max }^{\text {all }}$, so the whole building total electricity charges can be reduced. Meanwhile, in the $\left\{R_{L}, R_{M}, R_{H}\right\}$ and $\left\{R_{M}, R_{L}, R_{H}\right\}$ "rising unit price pattern" in which the final frame is the highest amount, there is no difference in Complex-RTP and Simple-RTP. This is because the mean room temperature deviation arrives at $R_{H}$ after falling, so it is difficult for the electricity charge reduction effect of Complex-RTP to be shown.

8.2 Complex Effect of the Total Room Temperature Deviation Regarding the total room temperature difference $T_{A D}^{p r i}$ of the priority block, the difference between Complex-RTP and Simple-RTP is within $+0.2\left[{ }^{\circ} \mathrm{C}\right]$, so almost the same level of room temperature comfort was shown. The difference between the two was at a maximum in the $\left\{R_{H}\right.$, $\left.R_{L}, R_{M}\right\}$ and $\left\{R_{H}, R_{M}, R_{L}\right\}$ "falling unit price pattern." In Complex-RTP, the room temperature of the priority air conditioner in the initial charge frame decreases by approximately $2\left[{ }^{\circ} \mathrm{C}\right]$. In subsequent frames, even air conditioners other than the prioritized one in which the temperature was still around $30\left[{ }^{\circ} \mathrm{C}\right]$ used power and decreased the room temperature. At this time, the whole building 5-minute power consumption is limited to $W_{5 \max }^{\text {all }}$, so the temperature decrease of the priority air conditioner in Complex-RTP occurs later than in SimpleRTP, causing a difference of $+0.2\left[{ }^{\circ} \mathrm{C}\right]$ in $T_{A D}^{p r i}$.

\section{Conclusion}

This report proposed a complex evaluation function for whole building air-conditioning power consumption and priority block room temperature in real-time pricing (RTP) of multi-unit building air conditioners. A simulation model (Aircon Emulator: AE) was built from an actual equipment test of RTP adjustment control in an actual office building, and the simulation which covered all unit price patterns was repeated, giving the following results.

(1) Complex RTP adjustment control reduced whole building power consumption by approximately $27 \%$ while providing the same level of room temperature comfort in the priority block as with the conventional simple RTP adjustment control.

(2) Between Complex-RTP adjustment control and SimpleRTP adjustment control, Complex-RTP reduced whole 
building electricity charges by a maximum of $33 \%$ in a manner dependent on the unit price pattern.

(3) Regarding the priority block room temperature, the difference between Complex-RTP adjustment control and Simple-RTP adjustment control was within $0.2^{\circ} \mathrm{C}$, so there was hardly a difference in room temperature comfort.

In the future, we will verify the effect when Complex-RTP adjustment control is applied to a large-sized building with approximately 20 outdoor units. At that time, the permutation pattern of the power limitation command value will increase explosively, so it will be difficult to derive a solution within the control time. Therefore, we plan to also study a means of reducing the calculation time by parallel computation.

\section{References}

( 1 ) C. Ninagawa: "Automated Demand Response Aggregation and Building Energy Management System”, Journal of IEIEJ, Vol.33, pp.40-43 (2013) (in Japanese)

( 2 ) C. Ninagawa: "Electric Power Markets and Automated Demand ResponseRealtime Pricing and System Stability-", Journal of IEIEJ, Vol.33, No.9, pp.3-6 (2013) (in Japanese)

( 3 ) H. Irie, A. Yokoyama, and Y. Tada: "System Frequency Control by Coordination of Batteries and Heat Pump based Water Heaters on Customer Side in Power System with a Large Penetration of Wind Power Generation", IEEJ Trans. on PE, Vol.130, No.3, pp.338-346 (2010) (in Japanese)

( 4 ) T. Masuta, K. Shimizu, and A. Yokoyama: "Load Frequency Control by use of a Number of Both Heat Pump Water Heaters and Electric Vehicles in Power System with a Large Integration of Renewable Energy Sources", IEEJ Trans. on PE, Vol.132, No.1, pp.23-33 (2012) (in Japanese)

( 5 ) S. Kawachi, H. Hagiwara, J. Baba, K. Furukawa, E. Shimoda, and S. Numata: "Study on Compensation of Short Term Power Fluctuation by Use of Heat Pump Air Conditioning System based on Real Machine", IEEJ Trans. on PE, Vol.132, No.1, pp.77-85 (2012) (in Japanese)

( 6 ) H. Kikuchi, H. Asano, and S. Bando: "Load Frequency Control by Commercial Air Conditioners Power Consumption Control with Large Penetration of Renewable Energy Generation”, IEEJ Trans. PE, Vol.135, No.4, pp.233-240 (2015) (in Japanese)

( 7 ) O. Ma, N. Alkadi, P. Cappers, P. Denholm, J. Dudley, S. Goli, M. Hummon, S. Kilcote, J. MacDonald, N. Matson, D. Olsen, C. Rose, M. Sohn, M. Starke, B. Kirby, and M. O'malley: "Demand Response for Ancillary Services”, IEEE Trans. on Smart Grid, Vol.4, No.4, pp.1988-1995 (2013)

( 8 ) H. Hao, Y. Lin, A.S. Kowli, P. Barooah, and S. Meyn: "Ancillary Service to the Grid Through Control of Fans in Commercial Building HVAC Systems", IEEE Trans. Smart Grid, Vol.5, No.4, pp.2066-2074 (2014)

( 9 ) Y. Lin, P. Barroah, S. Meyen, and T. Middlekoop: "Experimental Evaluation of Frequency Regulation From Commercial Building HVAC Systems", IEEE Trans. on Smart Grid, Vol.6, No.2, pp.776-783 (2015)

(10) K. Ma, G. Hu, and C. Spanos: "Distributed Energy Consumption Control via Real-Time Pricing Feedback in Smart Grid", IEEE Trans. on Control System Technology, Vol.22, No.5, pp.1907-1914 (2014)

(11) S. Noh, J. Yun, and K. Kim: "An Efficient Building Air-Conditioning System Control Under Real-Time Pricing", 2011 Int. Conf. on Advanced Power System Automation and Protection, pp.1283-1286 (2011)

(12) S. Ramdaspallli, M. Pipattanasomporn, M. Kuzlu, and S. Rahman: "Transactive Control for Efficient Operation of Commercial Buildings", IEEE Innovative Smart Grid Tchnologies Europe ISGTEurope, pp.1-5 (2016)

(13) J. Aguero, F. Rodriguez, M. Castilla, and M. Pereira: "Productiveness and Real Time Prices in Energy Management for HVAC Systems", 39th IEEE Conf. on Industrial Electronics IECON2013, pp.7956-7961 (2013)

(14) I. Murata and Y. Takarada: "Modeling of Dynamics in Demand Response for Real-Time Pricing", IEEE Int. Conf. on Smart Grid Communications, pp.806-811 (2014)

(15) S. Tamaru, D. Murayama, M. Murai, Y. Takagi, Y. Hanada, and K. Kimura: "The Energy Saving Control Method of Air Conditioing System with Outdoor Air Handling Units and Multi Air Conditioners for Buildings", 2013 IEEJ Annual Conf., Vol.4, pp.337-338 (2013) (in Japanese)

(16) N. Miyake, H. Takahashi, Y. Tomita, and K. Kuwagara: "Development of Cooperative Peak Cut Control Method for Building Electricity Demand with
Batteries and Facilities”, 2013 IEEJ Annual Conf., pp.31_7-31_8 (2013) (in Japanese)

(17) J. Morikawa, T. Yamaguchi, and C. Ninagawa: "Smart Grid Real-Time Pricing Optimization Management on Power Consumption of Building Multitype Air-Conditioners", IEEJ Trans. on Electrical and Electronic Engineering, Vol.11, No.6, pp.823-825 (2016)

(18) J. Morikawa and C. Ninagawa: "Fast Real-Time Pricing Optimization Control of Power Consumption and Room Temperature for Building Multi Type Air-Conditioning Facilities", IEEJ Trans. on PE, Vol.136, No.11, pp.817823 (2016) (in Japanese)

(19) H. Ito, C. Ninagawa, and J. Morikawa: "Fast Real-Time Pricing Optimization Control with Dynamic Prediction Model for Office Building Facilities", The papers of Technical Meeting on Smart Facilities, IEEJ, SMF-16-049, pp.7984 (2016) (in Japanese)

(20) S. Kirkpatrick, C. Gelatt, and M. Vecchi: "Optimization by Simulated Annealing", Science, Vol.220, No.4598, pp.671-680 (1983)

(21) E. Aarts and J. Korst: "Simulated Annealing and Boltzman Machines", John Wiley \& Sons, Chichester (1989)

(22) Y. Nagata, T. Naramura, C. Ninagawa, and J. Morikawa: "Short Time Prediction Model on Room Temperature Side-Effect due to Fast Demand Response of Building Multi Type Air-Conditioning Facilities Power Consumption”, 2016 IEEJ Annual Conference (2016) (in Japanese)

(23) T. Naramura, J. Morikawa, and C. Ninagawa: "Prediction Model on Room Temperature Side-Effect due to FastADR Aggregation for a Cluster of Building Air-Conditioning Facilities", IEEJ Trans. on PE, Vol.136, No.4, pp.432438 (2016) (in Japanese)

(24) T. Naramura, A. Shibata, J. Morikawa, and C. Ninagawa: "Prediction of Room Temperature Side-Effect due to Fast Demand Response for Building Air-conditioning Facilities", Int. Journal of Control, Communication and Systems, Vol.1, No.2, pp.11-19 (2016)

(25) T. Fukazawa, Y. Iwata, J. Morikawa, and C. Ninagawa: "Stabilization of Neural Network by Combination with AR Model in FastADR Control of Building Air-conditioner Facilities", IEEJ Trans. on Electrical and Electronic Engineering, Vol.11, No.1 (2016)

(26) S. Kondo, J. Morikawa, and C. Ninagawa: "Averaging Effect on Stochastic Response Prediction in FastADR Aggregation for Building Air-conditioning Facilities", IEEJ Transacitions on Electrical and Electronic Engineering, Vol.11, pp.820-822 (2016)

(27) T. Nakayama, A. Nakamura, C. Ninagawa, and J. Morikawa: "Deep Learning of Prediction Model on Building Air-Conditioning Power and Temperature Response for Smart Grid Fast Negative Watt Aggregation", Vol.4, pp.437238, 2017 IEEJ Annual Conference, Vol.4, pp.437-438 (2017)

(28) Y. Nagata, C. Ninagawa, and J. Morikawa: "Prediction Effect of Oil-Return Protection Operation on Real-Time Pricing Optimal Control of Building Multi-type Air-Conditioning Facilities", IEEJ Technical Meeting on Smart Facilities, SMF-17-004, pp.19-24 (2017) (in Japanese)

(29) T. Nohara and C. Ninagawa: "Study on Dynamic Characteristircs Simulation of Air-Conditioniners", Journal of JSME, Vol.C-53, pp.2115-2120 (1987) (in Japanese)

Yoshifumi Aoki (Non-member) received the B.Sc. degree in Mechan-

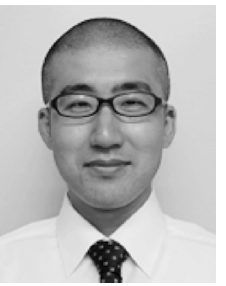
ical Engineering from Shizuoka University, Japan, in 2006. He has been with Mitsubishi Space Software Co., Ltd. from 2007 to 2014. He is now a researcher of Smart Grid Power Control Joint Research Lab., Faculty of Engineering, Gifu University, Japan, where he is currently pursuing the Ph.D. degree. His research interests include time series analysis for demand response.

Hiromichi Ito (Non-member) received the B.Sc. and the M.Sc. de-

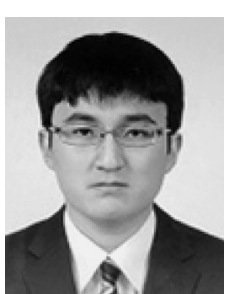
grees in Electric and Electrical Engineering from Gifu University, Japan, in 2016 and 2018, respectively. 
Chuzo Ninagawa (Senior Member) received the B.Sc., M.Sc., and

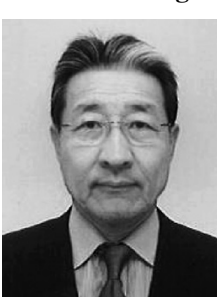
Ph.D. in Electrical Engineering from Nagoya University, Japan, and University of Washington, Seattle, U.S.A. He has been with Mitsubishi Heavy Industries, Ltd. from 1978 to 2012. He is now a professor of Smart Grid Power Control Joint Research Lab. Faculty of Engineering, Gifu University, Japan. His research interests include demand control for the future smart grid. He is a senior member of IEEJ, and also a member of IEEE, IEICE and IEIEJ.

Junji Morikawa (Non-member) received the M.Sc. degree from

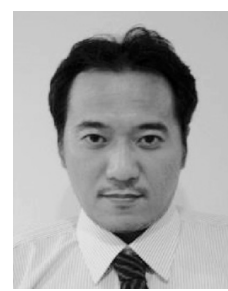
Nagoya Institute of Technology, Japan, in 2003 He joined Mitsubishi Heavy Industries, Ltd. in the same year. He is currently engaged in developing remote monitoring systems for building-multi airconditioning facilities in Mitsubishi Heavy Industries Thermal Systems, Ltd.
Takashi Inaba (Non-member) received the M.Sc. degree in Information Engineering from Nagoya University, Japan, in 1996. He joined Mitsubishi Heavy Industries, Ltd. in the same year. He is currently engaged in developing and designing electronic control systems for buildingmulti air-conditioners in Mitsubishi Heavy Industries Thermal Systems, Ltd.

Seiji Kondo (Non-member) received the B.Sc. degree in Electrical

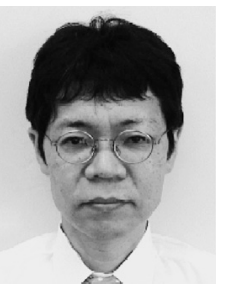
Engineering from Kyoto University, Japan, in 1992 He joined Mitsubishi Heavy Industries, Ltd. in the same year. He is currently engaged in researching and developing energy management systems for buildingmulti air-conditioning facilities in Mitsubishi Heavy Industries Thermal Systems, Ltd. 